\title{
Uso de Materiais Alternativos para Melhoria de Solo na Pavimentação de Vias
}

\section{Use of Alternative Materials for the Improvement of the Soil on Paving Roads}

\author{
${ }^{1}$ Leonardo Ramos da Silveira (Doutor em Geotecnia pela Universidade de Brasília., \\ leo_engambiental@hotmail.com); \\ ${ }^{2}$ Rafael de Assis Borges (Engenheiro Civil, eng.rafaelmiro@ hotmail.com).
}

\begin{abstract}
Recebido em março 2016 Aceito em maio 2016 Publicado em junho 2016
Resumo: O crescimento de obras no setor da construção civil faz com que aumente o uso de agregados naturais. Este grande uso, ocasiona em uma escassez em várias regiões, principalmente nas regiões de grande área ocupada, fazendo com que seja extraído o material natural em locais distantes do ponto de aplicação. Vários materiais alternativos como, resíduos de construção e demolição (RCD), resíduos de pedreira e fosfogesso, vêm sendo estudado a fim de avaliar o comportamento do solo com adições em várias proporções de material. $\mathrm{O}$ presente trabalho tem o intuito de apresentar uma discussão baseada em pesquisas já realizadas em grandes centros urbanos do Brasil, levando-se em conta a aplicação do material em camadas de pavimentação. São analisados os ensaios de caracterização do material e estudos de pistas experimentais. Com base nas pesquisas citadas, pode-se concluir que o aproveitamento de materiais alternativos como resíduos de pedreira, fosfogesso e $\mathrm{RCD}$, em camadas de pavimento, apresentam-se uma viabilidade técnica positiva.
\end{abstract}

Palavras Chave: resíduo; agregado reciclado; pavimentação; base; sub-base.

\begin{abstract}
The large growth of works in civil construction sector resulted on the increasing usage of natural aggregates. This great use of natural aggregates, leads to the lack of this substance in many other regions, especially in regions where large part of the substance is used, making necessary that the substance is extracted from distant places of the point of application. Several alternative materials as construction and demolition waste (CDW), and quarry wastes and phosphogypsum, have been studied in order to evaluate the behavior of the soil in various proportions of the material. This present work aims to introduce a discussion based in a research already produced in large urban centers in Brazil, considering the application of the material in paving layers. The material characterization tests and studies of experimental paving segment are analyzed. Based on these studies, it can be concluded that the use of alternative materials such as quarry waste, phosphogypsum and construction and demolition waste (CDW) in pavement layers, showing a positive technical feasibility.
\end{abstract}

Keywords: waste; recycled aggregate; paved; base; subbase.

\section{INTRODUÇÃO}

Atualmente, a geração de resíduos industriais tem tido destaque em função da sua capacidade de gerar problemas ambientais pelo seu descarte inadequado, devido à carência de alternativas técnicas e economicamente viáveis para o seu aproveitamento (LUZ, 2008). O acúmulo de resíduos gerados em qualquer atividade voltada para a qualidade da vida humana tornou-se um problema constante, como a maioria das tecnologias disponíveis ainda não são suficientes para tratar de maneira adequada ou eliminar estes resíduos. Encontrar métodos adequados de eliminação (sem afetar a qualidade do meio ambiente) é um tema importante no contexto atual, especialmente em grandes centros de pesquisa, que estudam materiais alternativos de baixo impacto ambiental (REZENDE et al., 2014).

De acordo com Pereira (2007), um dos maiores desafios ambientais enfrentados pela população mundial é a gestão eficaz de resíduos sólidos, devido a sua grande produção e diversidade, ficando cada vez mais difícil pensar em um destino ecologicamente correto e sustentável. A procura por materiais, a serem usados em camadas de base e sub-base que se adequem às especificações das normas 
rodoviárias vigentes no Brasil representam um grande problema para órgãos rodoviários locais tal qual é o problema da disposição e aproveitamento dos resíduos sólidos. Muitas vezes a falta de materiais granulares ou a sua grande distância do local de construção das vias acabam tornando inviável o uso em pavimentos. Dessa forma, surge a necessidade de utilizar solos menos nobres, estabilizandoos com aglomerantes e resíduos que melhorem suas propriedades.

Segundo Luz (2008), testar o aproveitamento do resíduo de pedreira e, por outro, sua aplicação à pavimentação rodoviária, é meta relevante na configuração tecnológica atual, visto que essa atividade absorve grandes quantidades de materiais para construção civil e pavimentação urbana, notadamente em regiões metropolitanas em franco crescimento. Além disso, esse aproveitamento do fíler pode contribuir para que se reduza a poluição por resíduos sólidos antes considerados inúteis, relacionados ao passivo ambiental, tornando-os uma espécie de "nova" matéria-prima, promovendo-os no status econômico e ambiental.

$$
\text { Para Canut (2006), o }
$$
reaproveitamento do resíduo fosfogesso, gerado na produção de ácido fosfórico a partir do beneficiamento de uma rocha fosfatada, é extremamente importante tanto do ponto de vista econômico-social quanto em relação à preservação ambiental, por se tratar de um resíduo abundante e cuja utilização poderá minimizar, ou até extinguir, a exploração de jazidas naturais de gesso.

A reciclagem de RCD como agregado para ser misturado ao solo na constituição das camadas de base, sub-base e revestimentos primários de pavimentação é a alternativa mais difundida e aceita no

\section{PROCEDIMENTOS METODOLOGICOS}

$\mathrm{O}$ presente artigo configura-se com base em um levantamento bibliográfico de meio técnico por possuir estudos mais consolidados (CABRAL e MOREIRA, 2011). No âmbito da construção civil, o alto crescimento de obras em todo território brasileiro tem feito com que aumente também a quantidade de entulho gerado. De acordo com Pinto (1999), esse entulho representa mais de $50 \%$ de todo o resíduo sólido urbano.

De acordo com Ribeiro (2006) o aproveitamento do entulho na engenharia civil pode representar economia, durabilidade e preservação ambiental, e a forma mais simples de reciclagem do entulho é a sua utilização em pavimentação, na forma de brita corrida ou ainda em misturas do resíduo com solo.

Levando em conta a necessidade dos recursos minerais para o desenvolvimento sócio-econômico, a dificuldade no controle da disponibilidade de insumos minerais coloca em risco, no presente, a manutenção e, para o futuro, a melhoria da qualidade de vida das populações. Os agregados para a indústria da construção civil são os insumos minerais mais consumidos e consequentemente os mais significativos em termos de volumes produzidos no Brasil e no mundo (SILVEIRA, 2010).

A escassez de jazidas naturais (solos e agregados) cujas características não se enquadram nas especificações técnicas tradicionais para o uso em pavimentação, aliada a uma legislação ambiental mais austera quanto à concessão de licenças para a exploração de jazidas naturais são fatores motivadores para a busca $\mathrm{e}$ utilização de materiais alternativos (BATALIONE, 2007). Sendo assim, esse trabalho trata-se de uma pesquisa bibliográfica para apresentar o uso de materiais alternativos visando sua aplicação em camadas de pavimento de vias.

artigos, dissertações e teses. $\mathrm{O}$ levantamento bibliográfico contempla 
sucinta discussão de três tipos de materiais alternativos que vêm sendo aplicados em camadas de base de pavimento com o intuito de melhoria das capacidades mecânicas do solo, como Índice de Suporte Califórnia, Resistência à Compressão

\section{RESULTADOS E DISCUSSÕES}

\section{Resíduos de pedreira}

O aumento do consumo de produtos cuja origem advém das atividades mineradoras vem proporcionando a toda população mundial um maior conforto (BATALIONE, 2007). A tendência de qualquer sistema natural é o aumento de seu grau de desordem quando não operado de forma adequada. Assim, utilizando os recursos naturais finitos e gerando energia de baixa qualidade, tendem a aumentar as desordens provenientes da má gestão do meio (SILVEIRA, 2010).

Segundo Luz (2008) em sua tese, considerando-se a problemática de busca de uma solução técnica alternativa para o aproveitamento de resíduo de pedreira de brita, o filer, partiu da hipótese de que sua mistura com solo laterítico local poderia ser viável e ao mesmo tempo importante para a questão da saúde da comunidade envolvida com o mesmo.

No trabalho de Rezende et al. (2014), foi desenvolvido um trecho experimental de $300 \mathrm{~m}$ de comprimento com base em resultados laboratoriais obtidos por Araújo (2008), localizado na região nordeste de Goiânia-GO. O pó de micaxisto usado na pesquisa é proveniente de uma pedreira localizada em Goiânia. O solo já existente no local foi compactado usando a energia Proctor normal e apresentou valores de 9\% no ensaio de índice de suporte Califórnia (ISC), que foi considerado como subleito. A incorporação do resíduo de pedreira foi feita em duas proporções: Mistura 1 (80\% de solo $+20 \%$ de pó de micaxisto) e Mistura $2(70 \%$ de solo $+30 \%$ de pó de micaxisto).
Simples, Módulo de Resiliência, etc. Em algumas pesquisas é citado o comportamento de trechos experimentais para comprovação de resultados obtidos em laboratório.

No ensaio de ISC, compactados nas energias Proctor intermediário e modificado, na Mistura 1, 40 e 53\%, e na Mistura 2, 27 e 43\%, respectivamente. Utilizou-se para a base a Mistura 1 e para sub-base a Mistura 2. No ensaio de viga Benkelman resultaram na prova de carga em $200 \mathrm{MPa}$ para a camada de base e subbase, indicando-se assim que, os valores determinados podem ser considerados adequados para estradas com baixos volumes de tráfego.

Batalione (2007) ensaiou um solo e rejeito de pedreira da região Noroeste do estado de Goiás, localizada a $35 \mathrm{~km}$ da capital do estado, nas cidades de Caturaí, Goianira e Inhumas. As misturas consistiram em proporções de 15, 25 e $35 \%$ de rejeito em relação ao peso do solo. A respeito da caracterização física dos materiais avaliados pôde-se concluir que a adição do rejeito aos solos naturais promoveu a redução do peso específico dos grãos de todas as amostras analisadas. Nos solos de Caturaí e de Inhumas os melhores desempenhos foram obtidos para a adição de $15 \%$ de rejeito em relação ao peso da amostra.

O solo de Goianira apresentou valores maiores de ISC em relação ao solo natural para todos os teores de rejeito, sendo verificado o melhor desempenho com a adição de $25 \%$. Esta quantidade de rejeito adicionada ao solo produziu um ISC de $20 \%$, credenciando esta mistura como material de sub-base, de acordo com a norma ES 301 (DNER, 1997). Para a pista experimental o autor relatou que se avaliados os resultados de todos os ensaios 
realizados em campo conclui-se que ambas as composições apresentaram índices muito próximos, demonstrando que a inclusão do rejeito fino de pedreira não prejudicou o desempenho do solo utilizado como material de base e nem da estrutura global do pavimento.

Silveira (2010), de acordo com o trabalho de Luz (2008) e Araújo (2008), monitorou-se o desempenho estrutural de um trecho de pavimento experimental e analisou misturas de solo com pó de micaxisto e solo com fíler. Separou-se as misturas em Mistura $1 \quad(30 \%$ fíler de micaxisto $+70 \%$ solo), Mistura $2(20 \%$ fíler de micaxisto $+80 \%$ solo), Mistura 3 $(20 \%$ pó de micaxisto $+80 \%$ solo), Mistura 4 (30\% pó de micaxisto $+70 \%$ solo). Fez-se análise química das misturas e do solo, resultando-se no ensaio de $\mathrm{pH}$ moderadamente ácido com valor máximo do solo e das Misturas 1 e 2, respectivamente, 5,5 e 6,8. Através de ensaios em laboratório resultou-se que:

a) Quimicamente, teve-se que a incorporação do fíler ao solo gera aumento de $\mathrm{pH}$, saturação de bases e capacidade de troca catiônica, o que pôde-se proporcionar um maior efeito de reação das bases trocáveis caso estivesse sido feito a estabilização química, o que poderia aumentar significativamente a resistência do material.

b) Mineralogicamente, nas misturas de solo com fíler prevaleceram-se os elementos existentes nos materiais de origem, sem a formação de novos compostos. Microscopicamente, o entrosamento dos materiais nas misturas depende principalmente da granulometria existente na mistura e da energia de compactação.

Ainda no estudo de Silveira (2010), no monitoramento do trecho experimental, de uma forma geral, o desempenho estrutural da via variou-se de regular a bom. Na superfície, observaram-se defeitos graves. No entanto, nas medidas dos deslocamentos foi-se possível identificar regiões com problemas. Esses problemas não estão diretamente relacionados com o tipo de material utilizado em cada subtrecho, mas sim com a topografia da pista e a inexistência de dispositivos de drenagem.

Luz (2008) analisou o fíler de uma pedreira localizada na Região Metropolitana de Goiânia-GO. Ensaiou misturas de $0,5,10,15,20,30,40,50$ e $100 \%$ de fíler no solo. Verificou-se que acima de $50 \%$ de fíler a mistura apresentou fraca coesão. No ensaio de compactação, os valores de máximo peso específico seco foram: para a proporção de $15 \%$ de fíler na energia Intermediário com valor de 18,15 $\mathrm{kN} / \mathrm{m}^{3}$; e, para a proporção de $20 \%$ de fíler na energia Modificado com valor de 18,9 $\mathrm{kN} / \mathrm{m}^{3}$. A partir dos resultados do ensaio de compactação, umidade ótima e máximo peso específico seco, as amostras para o ensaio de ISC foram moldadas. Usando como diretriz o valor de ISC, avaliaram-se as misturas para base e sub-base de acordo com o DNER (1996). Para a energia Intermediário numa proporção de $20 \%$ apresentou-se um valor maior e para energia Modificado numa proporção de $30 \%$ apresentou-se um valor maior.

Como soluções vislumbradas para conciliar este aumento da produção de bens que utilizam matéria prima mineral com as questões da preservação ambiental, pode-se destacar a busca e o uso de energias alternativas, melhorias no processo produtivo com redução do desperdício, e a substituição total ou parcial de matéria prima natural por materiais reciclados, ou de produtos que produzem menores impactos ao meio ambiente (BATALIONE, 2007). 
Fosfogesso é o nome dado ao subproduto da reação química da rocha fosfática com ácido sulfúrico e água. Por meio desta reação, obtém-se o ácido fosfórico, que é usado, principalmente, como matéria-prima para a produção de fertilizantes fosfatados (MESQUITA, 2007).

A sua denominação, fosfogesso, indica sua origem industrial (fosfo) e seu componente principal (gesso). Esse subproduto é sulfato de cálcio $\left(\mathrm{CaSO}_{4}\right)$ e, portanto, apresenta a mesma composição que o gesso natural (OLIVEIRA, 2005).

Rufo (2009) ao estudar as misturas de fosfogesso, solo e cal para fins de pavimentação, realizou um estudo laboratorial onde as misturas foram denominadas Misturas $1(90 \%$ solo $+10 \%$ fosfogesso), Misturas 2 (80\% solo $+20 \%$ fosfogesso), Misturas 3 (70\% solo $+30 \%$ fosfogesso), Misturas 4 (97\% solo $+3 \%$ cal calcítica CHIII), Misturas 5 (94\% solo $+6 \%$ cal calcítica CHIII), Misturas $6(91 \%$ solo $+9 \%$ cal calcítica CHIII), Mistura 7 $(81,9 \%$ solo Catalão-GO $+9,1 \%$ fosfogesso $+9 \%$ cal CHIII), Mistura 8 $(81,9 \%$ solo Catalão-GO $+9,1 \%$ fosfogesso + 9\% cal dolomítica), Mistura 9 $(84,6 \%$ solo Goiânia-GO $+9,4 \%$ fosfogesso $+6 \% \mathrm{Cal} \mathrm{CHI)}$.

Nas misturas de solo-fosfogesso estudadas por Rufo (2009), verificou-se que com os teores de $10 \%$ e $20 \%$ houve ganho de resistência do solo, sendo possível a utilização das Misturas 1 e 2 em sub-base de pavimentos. Para as misturas de solo-cal (Misturas 4, 5 e 6) foi constatado que o ISC aumentou com a adição de cal, sendo que para teor de cal superior a $6 \%$ o ganho no valor de ISC foi maior. $\mathrm{O}$ ensaio de resistência à compressão simples mostrou que ocorre um ganho no valor da resistência do solo quando a cal é utilizada e decréscimo quando o fosfogesso é utilizado.

As Misturas 7 e 8 apresentaram problemas relacionados à expansão elevada. Além disso, os valores de CBR encontrados foram muito baixos, $2,1 \%$ para a Mistura 7 e 3,3\% para a Mistura 8, indicando assim que estas misturas não podem ser utilizadas para construção de pavimentos. A Mistura 9 apresentou valor de CBR satisfatório (superior a 60\%), porém a expansão foi superior a $2 \%$ fazendo-se necessário uma correção na expansão para a viabilidade desta mistura.

Mesquita (2007) estudou a aplicação de fosfogesso em um solo da região de Goiânia-GO. As amostras ensaiadas foram divididas em: solo, Mistura A $(50 \%$ de solo local $+50 \%$ fosfogesso), Mistura B ( $80 \%$ de solo local $+20 \%$ fosfogesso), Mistura C $(20 \%$ de solo local $+80 \%$ fosfogesso) e fosfogesso. Observou-se que, quando compactados, quanto maior a quantidade de fosfogesso presente na mistura, menor é o valor do peso específico aparente seco máximo (em torno de $12 \mathrm{KN} / \mathrm{m}^{3}$ ) e maior é o teor de umidade ótima (superior a $30 \%$ ).

Ainda segundo Mesquita (2007), verificou-se que para a Mistura B (20\% de fosfogesso) apresentou maior potencial de aplicação, podendo ser utilizada em camadas de sub-base de pavimentos (ISC = $37 \%)$. A Mistura C (80\% de fosfogesso) não seria indicada para uma aplicação prática, pois apresentou capacidade de suporte baixa e semelhante aos solos normalmente encontrados nos subleitos brasileiros (ISC $=7,0 \%$ ). $\mathrm{O}$ solo e a Mistura A (50\% de fosfogesso) poderiam ser utilizados no máximo como reforço do subleito ou na construção de aterros em geral $($ ISC $=15 \%)$.

Metogo (2011) em seu trabalho avaliou-se o comportamento mecânico em campo de uma pista experimental executada com misturas de solo-cal, solofosfogesso e solo-fosfogesso-cal em sua camada de base $(15 \mathrm{~cm})$. Sua pesquisa teve base nos resultados obtidos nos trabalhos realizados por Mesquita (2007), Faria (2007) e Rufo (2009). Como resultado, no ensaio de compactação, analisando-se a posição do Eixo, observou-se valores de $112 \%$ solo-fosfogesso, $96 \%$ solofosfogesso-cal, $101 \%$ solo-cal, para o grau 
de compactação. No ensaio de índice de suporte Califórnia (ISC) as misturas apresentaram valores de $24 \%$ solofosfogesso, $44 \%$ solo-cal e $88 \%$ solofosfogesso-cal.

Oliveira (2005), para sua pesquisa, coletou o fosfogesso em seu estado natural, em um depósito que pertence a uma indústria de fertilizantes, localizada no município de Uberaba-MG. A preparação do fosfogesso consistiu em sua secagem ao ar livre até se atingir a umidade higroscópica. Em seguida, o material foi homogeneizado com operações de destorroamento, passagem na peneira $\mathrm{n}^{\mathrm{o}} 4$ $(4,75 \mathrm{~mm})$, e posterior quarteamento.

No programa experimental de Oliveira (2005), analisaram-se as seguintes

\section{Resíduos da construção e demolição (RCD)}

A incorporação do agregado reciclado no solo para uso em camadas de pavimentos tem demonstrado resultados significativos em várias pesquisas realizadas em vários grandes centros urbanos (FUJII, 2012; GÓMEZ, 2011; LEITE，2007; QUINTANILHA， 2008; MOTTA, 2005; SANTOS, 2007; RESPLANDES, 2007; RIBEIRO, 2006). Em sua maioria, suas pesquisas para aplicação em camadas de pavimentos indicaram índices de suporte Califórnia (ISC) satisfatórios de acordo com a NBR 15115 (ABNT, 2004).

Algumas peculiaridades foram descritas em função de outras características físicas da mistura soloRCD. Leite (2007) ressaltou sobre a influência da energia de compactação em seus resultados, fazendo com que variasse de $73 \%$ na energia de compactação Intermediário e $117 \%$ na energia de compactação Avançado no resultado do ISC do agregado reciclado.

Santos (2007), nos ensaios de limite de consistência revelaram que todas as amostras ensaiadas possuem $\mathrm{o}$ comportamento não plástico, tal resultado pode ser justificado pela análise misturas: $0 \%$ de cal e $100 \%$ de fosfogesso, Mistura $1 \quad(3 \%$ de cal e $97 \%$ de fosfogesso), Mistura 2 (5\% de cal e $95 \%$ de fosfogesso), Mistura 3 (7\% de cal e 93\% de fosfogesso) e Mistura 4 (10\% de cal e $90 \%$ de fosfogesso). Os corpos-deprova foram compactados na energia equivalente ao Proctor modificado. Verificou-se que o tempo de cura foi um fator importante no desenvolvimento da resistência devido à presença de cal nas misturas. Em relação ao ensaio de ISC, o fosfogesso puro é muito suscetível à ação da água, apresentando valor igual a $16 \%$. A adição de 3\%, 5\%, 7\% e 10\% de cal conduz a valores de ISC de 54\%, 56\%, $65 \%$ e $71 \%$, respectivamente.

microscópica realizada com a fração passante na peneira de abertura $0,075 \mathrm{~mm}$, onde se verificou a presença de partículas de material cimentício. Observou-se que o ensaio de compactação não provocou significativamente a quebra de partículas. $E$ que o ensaio de ISC revelou que o agregado reciclado ensaiado trata-se de um material não expansivo, com valor de ISC igual a $60 \%$.

Quintanilha (2008) mostrou, em sua pesquisa, que a quantificação das fases cimentícias e cerâmicas influenciam sobremaneira sobre as propriedades de resistência. Separaram-se as amostras em função da composição granulométrica dos materiais em proporções de $25 \%$ para cada material, sendo: Amostra 1 ACR1 agregado reciclado composto por elementos cimentícios; Amostra 2 ARM1 agregado reciclado composto por elementos cimentícios e cerâmicos (19,0 $\mathrm{mm}, 9,5 \mathrm{~mm}, 4,8 \mathrm{~mm}$ e solo argiloso para amostras 1 e 2); Amostra 3 NAT1 material natural extraído de jazida; Amostra 4 ARC2 material reciclado composto por elementos cimentícios (retirou-se $\mathrm{O}$ material 4,8 mm das amostra 1 e 2, e usouse pó de brita nas amostras 3 e 4); e, 
Amostra 5 solo argiloso. Em todas as dosagens apresentaram resultados satisfatórios de resistência, medidas pelo ensaio de ISC. Nas pistas experimentais apresentaram bom comportamento estrutural, comprovando tecnicamente a viabilidade da construção de vias urbanas, preferencialmente de baixo volume de tráfego.

Gómez (2011), em sua pesquisa, ensaiou o agregado reciclado oriundo da demolição do Estádio Mané Garrincha em Brasília/DF. No conhecimento dos materiais constituintes do RCD, foram selecionados $13 \mathrm{~kg}$ de material seco os quais foram misturados para homogeneizar a amostra e posteriormente submetidos a peneiramento visando separação dos tamanhos graúdos $\left(1 "-\mathrm{N}^{\circ} 4\right)$ e miúdos $\left(\mathrm{N}^{\circ}\right.$ 4 - fundo). Separando o material por tamanho, resultou-se na classificação visual em RCD (99,43\%), madeira $(0,37 \%)$, plástico $(0,18 \%)$ e metais $(0,02 \%)$. Os materiais que passaram na peneira $\mathrm{N}^{\mathrm{o}} 4(75 \mathrm{~mm})$ foram classificados como finos e não foi possível a separação manual desses. Segundo a NBR 15115 (ABNT, 2004), o resultado obtido $(\mathrm{RCD}=$ $99,43 \%$ ) para a amostra total se enquadrou no valor especificado de RCD > 97\%.

Nos ensaios mecânicos, verificou-se índices de suporte Califórnia satisfatórios, superando o limite da norma NBR 15115 (ABNT, 2004) de 60\%. Os resultados dos ensaios de Módulo de Resiliência mostraram que o agregado reciclado pesquisado tem comportamento similar ao de agregados pétreo comumente utilizados, apresentando valores variando entre 200 e $500 \mathrm{MPa}$, e com sete dias de cura variaram entre 300 e $600 \mathrm{MPa}$.

Motta (2005), através de ensaios realizados, mostraram que grande parte do

\section{CONSIDERAÇÕES FINAIS}

Com base nas pesquisas citadas, pode-se concluir que o aproveitamento de materiais alternativos como resíduos de pedreira, fosfogesso, resíduo da construção agregado reciclado é composta de materiais cimentícios - concreto e argamassa - e naturais britados. Verificouse que o agregado reciclado em geral absorve muita água, que certamente é explicado devido à sua porosidade. O ISC aumentou significativamente com o tempo, explicando-se pela presença de atividade pozolânica no agregado reciclado, que foi ativado pela compactação, aumentando significativamente a quantidade de finos, aliada à adição de água, provocando reação pozolânica com o tempo. Os valores encontrados no ensaio de ISC do agregado reciclado após cura de 90 e 180 dias, compactados na energia Proctor Intermediária, foram de $117 \%$ e $125 \%$, respectivamente.

Resplandes (2007) separou três amostras (L1, L2 e L3) em função da faixa granulométrica para a pesquisa, sendo: L1 brita $19 \mathrm{~mm}$; L2 brita $9,5 \mathrm{~mm}$; e L3 brita $4,8 \mathrm{~mm}$. Concluiu-se que com os resultados obtidos a partir de ensaios de laboratório teve-se que a amostra L1 não apresentou melhores resultados nos ensaios de característica mecânica, como o índice de suporte Califórnia, podendo ser explicado pela falta de finos na mistura, o que gera inexistência de coesão no material. Após 15 dias de cura, as outras duas amostras ultrapassaram a marca dos $100 \%$, indicando que o agregado reciclado estudado, possui uma capacidade de suporte maior do que a da brita-padrão.

$\mathrm{Na}$ pesquisa de Resplandes (2007), ressaltou-se também que em alguns ensaios de campo, observou que há melhoria das propriedades medidas ao longo do tempo, o que pode ser explicado pelas reações pozolânicas que continuam ocorrendo neste tipo de material.

e demolição (RCD), em camadas de pavimento, apresentam-se uma viabilidade técnica positiva, atentando-se para um bom controle de proporção da mistura 
(solo-resíduo) de aplicação e de execução, é possível ter um pavimento com ótimo desempenho.

Ambientalmente, o uso desses resíduos é extremamente benéfico ao meio ambiente uma vez que esses materiais, geralmente, são descartados e dispostos de forma inadequada. A grande exploração de jazidas naturais causa sua escassez por se tratar de uma fonte não renovável e finita. Grandes centros urbanos estão aumentando o custo de suas obras devido

\section{REFERÊNCIAS BIBLIOGRÁFICAS}

AMORIM, E. F. Viabilidade Técnica Econômica de Misturas de Solo-RCD em Camadas de Base de Pavimentos Urbanos. Estudo de Caso: Município de Campo Verde - MT. 2013. 151 f. Tese (Doutorado em Geotecnia) - Universidade de Brasília, Brasília, 2013.

ARAÚJO, W. E. L. Aproveitamento de resíduos da extração de micaxisto em pavimentos flexíveis. 2008. $142 \mathrm{f}$. Dissertação (Mestrado em Recursos Hídricos e Saneamento Ambiental) Universidade Federal de Goiás, Escola de Engenharia Civil, Goiânia, 2008.

ASSOCIAÇÃO BRASILEIRA DE NORMAS TÉCNICAS. NBR 15115/2004: Agregados reciclados de resíduos sólidos da construção civil - Execução de camadas de pavimentação Procedimentos. Rio de Janeiro, 2004.

BATALIONE, G. Estabilização de solos tropicais com a utilização de rejeitos finos de pedreira de uma rocha granítica. 2007, 172 f. Dissertação (Mestrado em Geotecnia) - Universidade de Brasília, Brasília, 2007.

BRASIL CONSELHO NACIONAL DO MEIO AMBIENTE - CONAMA Resolução N 307, de 5 de Julho de 2002. às grandes distâncias de transporte desse material até seu ponto de aplicação, aumentando o custo do transporte.

A difusão de pesquisas nessa área, desperta à sociedade para estar sempre buscando tecnologias novas e cada vez mais ecológicas e sustentáveis. A população em geral é prejudicada pelos grandes volumes de resíduos acumulados em indústrias ou em áreas das cidades, causando poeira, poluição visual etc.

Ministério do Meio Ambiente, Conselho Nacional do Meio Ambiente, 2002.

CABRAL, A. E. B.; MOREIRA, K. M. V. Manual sobre os resíduos sólidos da construção civil - Sinduscon/CE. (Sindicato da Indústria da Construção Civil do Ceará). 2011. 44 f. Programa Qualidade de Vida na Construção. Fortaleza, CE, Agosto de 2011.

CANUT, M. M. C. Estudo da viabilidade do uso do resíduo fosfogesso como material de construção. 2006, 154 f. Dissertação (Mestrado em Construção Civil) - Escola de Engenharia da Universidade Federal de Minas Gerais, Belo Horizonte, 2006.

CARNEIRO et al. Reciclagem de entulho para produção de materiais de construção. Organizado por Alex Pires Carneiro, Irineu Antônio Schadach de Brum e José Clodoaldo da Silva Cassa. Salvador: EDUFBA; Caixa Econômica Federal, 312 p. 2001.

DEPARTAMENTO NACIONAL DE ESTRADAS DE RODAGEM - DNER. Método de dimensionamento de pavimento flexível. Rio de Janeiro, RJ, 1996. 
DNER (1997a). ES-301

Pavimentação - Sub-base estabilizada granulometricamente. DNIT/DNER, Rio de Janeiro, RJ, 7 p. 1997.

FARIA, L. F. Ensaios Laboratoriais com mistura de solo, fosfogesso e cal. Projeto de Iniciação Científica UFG. 2007.

FUJII, L. M. T. Estudo de misturas de solo, RCD e cal virgem e hidratada para uso em obras rodoviárias. 2012. 137 f. Dissertação (Mestrado em Geotecnia) Universidade de Brasília, Brasília, 2012.

GÓMEZ, A. M. J. Estudo experimental de um resíduo de construção e demolição (RCD) para utilização em pavimentação. 2011. 123 f. Dissertação (Mestrado em Geotecnia) - Universidade de Brasília, Brasília, 2011.

LEITE, F. C. Comportamento mecânico de agregado reciclado de resíduo sólido da construção civil em camadas de base e sub-base de pavimentos. 2007. $185 \mathrm{f}$. Dissertação (Mestrado em Engenharia de Transporte) - Escola Politécnica da Universidade de São Paulo, São Paulo, 2007.

LUZ, M. P. Aproveitamento de filer de pedreiras da região metropolitana de Goiânia em pavimentos flexíveis urbanos: avaliação técnica e sócioambiental. 2008. 103 f. Tese (Doutorado em Ciências Ambientais) - Universidade Federal de Goiás, Goiânia, 2008.

MESQUITA, G. M. Aplicação de misturas de fosfogesso e solos tropicais finos na pavimentação. 2007. 144 f. Dissertação (Mestrado em Recursos Hídricos e Saneamento Ambiental) Universidade Federal de Goiás, Escola de Engenharia Civil, Goiânia, 2007.

Metogo, D. A. N. Construção e avaliação inicial de um trecho de pavimento asfáltico executado com misturas de solo tropical, fosfogesso e cal. 2010. 196 f. Dissertação (Mestrado em
Geotecnia) - Universidade Federal de Goiás, Escola de Engenharia Civil, Goiânia, 2010.

MOTTA, R. S. Estudo laboratorial de agregado reciclado de resíduo sólido da construção civil para aplicação em pavimentação de baixo volume de tráfego. 2005. 134 f. Dissertação (Mestrado em Engenharia de Transporte) Escola Politécnica da Universidade de São Paulo, São Paulo, 2005.

OLIVEIRA, S. M. F. Estudo do comportamento mecânico de misturas de fosfogesso e cal para utilização na construção rodoviária. 2005.200 f. Dissertação (Mestrado em Transporte) Escola de Engenharia de São Carlos da Universidade de São Paulo, São Paulo, 2005.

PEREIRA, K. L. A. Estabilização de um solo com cimento e cinza de lodo para uso em pavimentos. 2012. $125 \mathrm{f}$. Dissertação (Mestrado em Engenharia Civil) - Universidade Federal do Rio Grande do Norte. Centro de Tecnologia. Natal, 2012.

PINTO, T. P. Metodologia para a gestão diferenciada de resíduos sólidos da construção urbana. 1999, 189 f. Tese (Doutorado em Engenharia de Construção Civil e Urbana) - Escola Politécnica da Universidade de São Paulo, São Paulo, 1999.

QUINTANILHA, F. S. Avaliação de utilização e desempenho de resíduos de construção e demolição em bases e subbases de pavimentos flexíveis. 2008. 114 f. Dissertação (Mestrado em Recursos Hídricos e Saneamento Ambiental) Universidade Federal de Goiás, Escola de Engenharia Civil, Goiânia, 2008.

RESPLANDES, H. M. S. Estudo ambiental e mecânico da aplicação do agregado reciclado na estrutura de pavimentos flexíveis. 2007. $206 \mathrm{f}$. 
Dissertação (Mestrado em Recursos Hídricos e Saneamento Ambiental) Universidade Federal de Goiás, Escola de Engenharia Civil, Goiânia, 2007.

REZENDE, L. R.; SILVEIRA, L. R.; ARAÚJO, W. R.; LUZ, M. P. Reuse of Fine Quarry Wastes in Pavement: Case Study in Brazil. Journal of Materials in Civil Engineering. Vol. 26, № 8, August 2014.

RIBEIRO, F. Estudo da aplicação do agregado reciclado na base de um pavimento flexível. 2006. $171 \mathrm{f}$. Dissertação (Mestrado em Recursos Hídricos e Saneamento Ambiental) Universidade Federal de Goiás, Escola de Engenharia Civil, Goiânia, 2006.

ROCHA, E. G. A. Os Resíduos Sólidos de Construção e Demolição: gerenciamento, quantificação e caracterização. Um estudo de caso no Distrito Federal. 2006. 155p. (Mestre em Estruturas e Construção Civil) Universidade de Brasília, Brasília, 2006.

RUFO, R. C. Estudo laboratorial de misturas de fosfogesso, solo tropical e cal para fins de pavimentação. 2009. $153 \mathrm{f}$. Dissertação (Mestrado em Recursos Hídricos e Saneamento Ambiental) Universidade Federal de Goiás, Escola de Engenharia Civil, Goiânia, 2009.

SANTOS, E. C. G. Aplicação de resíduos da construção e demolição reciclados (RCD-R) em estruturas de solo reforçado. 2007. 168 f. Dissertação (Mestrado em Geotecnia) - Escola de Engenharia de São Carlos da Universidade de São Paulo, São Paulo, 2007.

SILVEIRA, L. R. Reaproveitamento de finos de pedreiras em pavimentação: uma abordagem técnica e econômica. 2010. 179 f. Dissertação (Mestrado em Recursos Hídricos e Saneamento Ambiental) - Universidade Federal de
Goiás, Escola de Engenharia Civil, Goiânia, 2010. 